EPJ Web of Conferences 66, 06015 (2014)

DOI: $10.1051 /$ epjconf/ 20146606015

(C) Owned by the authors, published by EDP Sciences, 2014

\title{
Studies of the transverse structure of the nucleon at JLab
}

\author{
Marco Mirazita ${ }^{1, a}$ \\ ${ }^{1}$ I.N.F.N. - Laboratori Nazionali di Frascati
}

\begin{abstract}
An overview of the studies on the Trasverse Momentum Dependent parton distribution functions performed at the Thomas Jefferson Laboratory in the three experimental Halls with the $6 \mathrm{GeV}$ electron beam will be presented. Plans for the future measurements after the upgrade of the laboratory currently underway will be also discussed.
\end{abstract}

\section{Introduction}

For many years, the internal structure of the nucleon has been studied through Deep Inelastic Scattering (DIS) experiments performed in different kinematic regimes. Based on these large amount of data in a domain that spans several order of magnitude in both the four-momentum transfer $Q^{2}$ and the Bjorken variable $x_{B}$, we have nowadays a good knowledge of the longitudinal distributions of the momentum and spin of the quarks inside the nucleon, where longitudinal refers to the direction parallel to that of the exchanged hard virtual photon. Information on the mono-dimensional nucleon structure is encoded in two Parton Distributions Functions (PDFs), that are functions of the partonic longitudinal momentum:the unpolarized PDF $f_{1}\left(x_{B}\right)$ and the helicity PDF $g_{1}\left(x_{B}\right)$.

At leading twist, the description of the nucleon structure is completed by a third PDF, the transversity distribution $h_{1}\left(x_{B}\right)$, describing the contribution of transversely polarized quarks to a transversely polarized nucleon. Being chiral-odd, the transversity can only be measured associated to another chiral-odd object, thus it is not accessible in inclusive DIS experiments. For long time, transverse spin effects have been considered to be small. It took some time to realize that unsuppressed transverse spin effects may contribute to explain the surprisingly large and otherwise puzzling Single-Spin Asymmetries (SSA) observed in hadronic reactions with transversely polarized protons.

The Semi-Inclusive Deep Inelastic Scattering (SIDIS) processes have been identified as a powerful tool to explore transverse effects. In this processes, a final hadron is detected in coincidence with the scattered electron, with the advantage of providing unique information on the flavor of the quark involved in the scattering process ("flavor tagging") and of extending the study of the nucleon structure in a three-dimensional space. In fact, when the transverse momentum of the quarks $k_{\perp}$ is not integrated out, a variety of new PDFs emerges, describing the correlations between the quark or nucleon spin with the quark transverse momentum ("spin-orbit correlations"). For this reason, these new functions are called Transverse Momentum Dependent (TMDs) partonic distribution functions.

\footnotetext{
a e-mail: marco.mirazita@lnf.infn.it
} 


\section{TMDs in SIDIS processes}

The rigorous basis for studying TMDs in SIDIS processes has been provided by factorization in QCD, which has been established [2-5] for leading-twist single hadron production from a quark with transverse momentum $k_{\perp}$ smaller than the hard scale $Q^{2}$.

At leading-twist, there are eight TMDs, each of them having a specific probabilistic interpretation as quark number densities. Three of them are three-dimensional generalizations of the PDFs measured in inclusive DIS. The other five are new objects, vanishing upon integration over the quark transverse momentum. All of them enter in the SIDIS cross section through structure functions (18 at leading twist), that are convolution integrals, over the quark momenta, with a Fragmentation Function (FF) [1] that describes the hadronization of the scattered quark into the final hadron. In the case of an unpolarized final hadron, the FF contributing to the SIDIS process may be either the unpolarized $D_{1}$ or the Collins $H_{1}^{\perp}$ [11] function.

The various structure functions may be extracted by measuring beam and/or target spin asymmetries. Data on single and double spin asymmetries have been reported in single pion and kaon SIDIS production by the HERMES [6-9] and COMPASS [10] Collaborations and by the CLAS, Hall A and Hall C experiments of the Thomas Jefferson Laboratory (JLab).

\section{TMD results at JLab}

A significant part of the physics program of the JLab running with the $6 \mathrm{GeV}$ electron beam has been devoted to the studies of TMDs. Hall A and Hall C host small acceptance spectrometers for high-precision measurements, while the CLAS in Hall B is a $4 \pi$ detector for measurements in a wide kinematic range. Data have been collected using different types of unpolarized or polarized targets, including a transversely polarized ${ }^{3} \mathrm{He}$ target installed in Hall A to study TMDs of the neutron. This allowed the measurement of a variety of different single or double spin asymmetries (see figure 1 for some selected results) from which, together with results from other experiments like Hermes and Compass, important conclusions may be drawn.

There are indications that all the eight leading twist TMDs are non-zero. Of particular interest is the Sivers [12] function, entering in the transverse target spin asymmetry, because of its link to the quark orbital angular momentum [13], the main still unmeasured contribution to the nucleon spin. Measurements of multiplicities and double spin asymmetries as a function of the final transverse momentum of pions $[14,15]$ suggest that transverse momentum distributions may depend on the polarization of quarks and possibly also on their flavor. This is also pointed out by the different behavior of the Collins and Sivers asymmetries measured on the neutron [16] with respect to the proton results from Hermes [17, 18].

JLab reported also measurements of non-zero higher twist effects, as for example in the the single pion Beam [19, 20] and Target Spin Asymmetry [15]. Higher twist observables are important for understanding the long-range quark-gluon dynamics and they are accessible as leading contributions [21-23]. Recently, higher twist effects in SIDIS were interpreted in terms of average transverse forces acting on the active quarks in the instant after being struck by the virtual photon [24].

\section{Future TMD measurements at JLab}

The measurement of SIDIS observables is one of the driving physics items of the, currently underway, upgrade of the JLab. The maximum beam energy will be increased to $12 \mathrm{GeV}$ and the experimental equipments of the existing Halls will be upgraded. In particular, the capabilities of the Hall A and C 


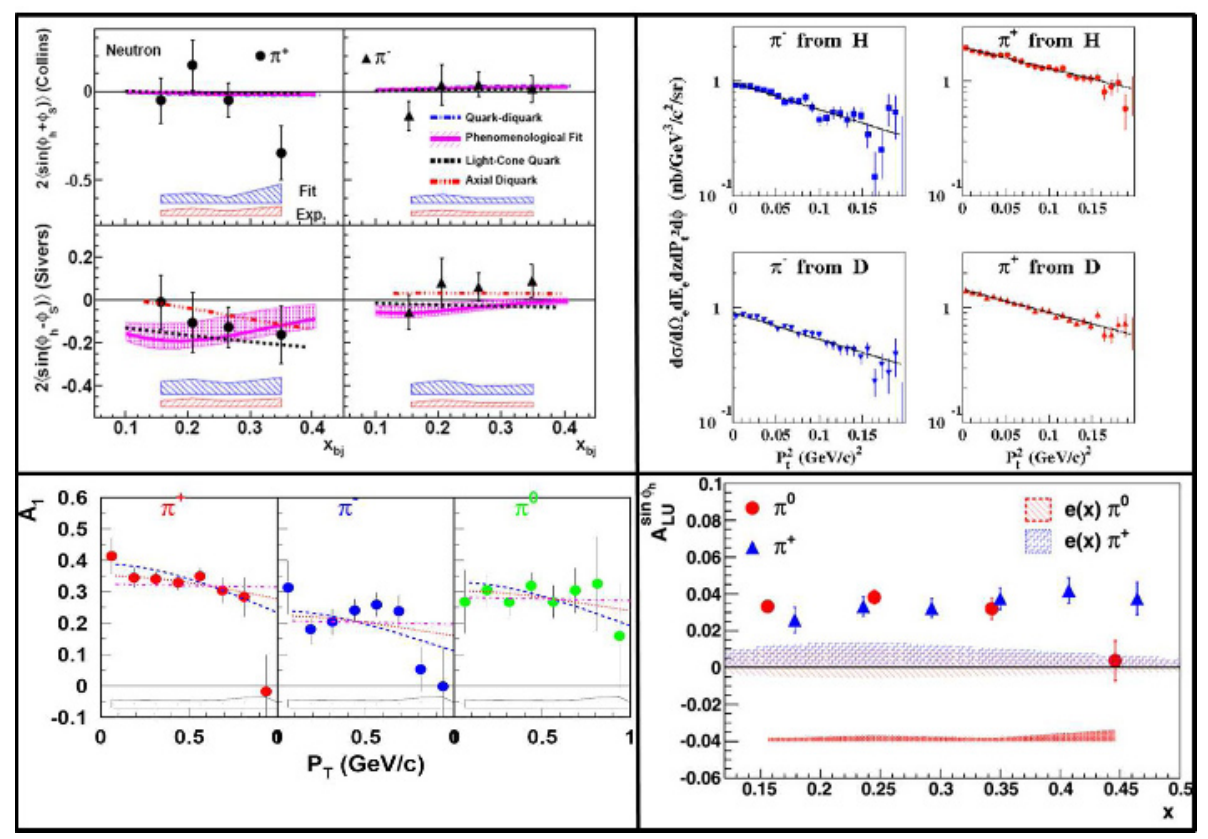

Figure 1. Selected TMD results from JLab. Top left panel: Hall A measurements [16] of charged pions Collins and Sivers asymmetries on the neutron; top right panel: Hall C measurements [14] of the charged pions unpolarized cross section on proton and deuteron. bottom left panel: CLAS measurement [15] of the double spin asymetries for pions. bottom right panel: CLAS measurement [19] of the BSA for pions. For the description of the curves, refer to the quoted original papers.

spectrometers will be improved with more refined tracking and particle ID devices. In Hall B, the new CLAS12 large acceptance spectrometer will be built. The implementation of a RICH detector in the CLAS12 is also under development, in order to extend the SIDIS measurements in the strange sector and to allow a full TMD flavor separation. Finally, the SOLID project foresees the installation in Hall A of a completely new large acceptance spectrometer with detection characterstics complemetary to CLAS12.

In the $12 \mathrm{GeV}$ era, JLab will produce an enormous amount of data, order of magnitudes bigger than what is available now, allowing the study of the TMDs with an unprecedented precision, epsecially in the valence (i.e. high- $x_{B}$ ) region. This calls for the elaboration of new multidimensional analysis techniques that are currently under development using both experimental and simulated data.

\section{References}

[1] A. Bacchetta et al., JHEP 02, 093 (2007)

[2] X.-d. Ji, J.-p. Ma, and F. Yuan, Phys. Rev. D 71 (2005) 034005, hep-ph/0404183.

[3] J. C. Collins and A. Metz, Phys. Rev. Lett. 93 (2004) 252001, hep-ph/0408249

[4] A. Bacchetta, D. Boer, M. Diehl, and P. J. Mulders, JHEP 08 (2008) 023, arXiv:0803.0227 [hep$\mathrm{ph}]$.

[5] J. Collins, T. Rogers, and A. Stasto, Phys.Rev. D77 (2008) 085009, arXiv:0708.2833 [hep-ph]. 
[6] A. Airapetian, et al., Phys. Rev. Lett. 84, 4047-4051 (2000), hep-ex/9910062

[7] A. Airapetian, et al., Phys. Rev. D64, 097101 (2001), hep-ex/0104005.

[8] A. Airapetian, et al., Phys. Rev. Lett. 94, 012002 (2005), hep-ex/0408013

[9] A. Airapetian, et al., Phys. Lett. B648, 164-170 (2007), hep-ex/0612059

[10] V. Y. Alexakhin, et al., Phys. Rev. Lett. 94, 202002 (2005), hep-ex/0503002

[11] J. C. Collins, Phys. Lett. B536, 43-48 (2002), hep-ph/0204004

[12] D. W. Sivers, Phys. Rev. D 41, 83 (1990).

[13] S. J. Brodsky, D. S. Hwang, and I. Schmidt, Phys. Lett. B 530, 99 (2002).

[14] H. Mkrtchyan, et al., Phys. Lett. B665, 20-25 (2008), 0709.3020.

[15] H. Avakian et al., Phys. Rev. Lett. 105 (2010) 262002, arXiv:1003.4549 [hep-ex].

[16] X. Qian et al. (JLab HALL-A), Phys. Rev. Lett. 107, 072003 (2011).

[17] A. Airapetian et al., Phys. Rev. Lett. 103 (2009) 152002, 0906.3918.

[18] A. Airapetian et al., Phys. Lett. B693 (2010) 11, 1006.4221.

[19] M. Aghasyan et al., Phys.Lett. B704, 397 (2011), 1106.2293.

[20] H. Avakian et al., Phys. Rev. D69, 112004 (2004), hep-ex/0301005.

[21] R. D. Tangerman, and P. J. Mulders, Phys. Rev. D51, 3357-3372 (1995), hep-ph/9403227

[22] A. Kotzinian, Nucl. Phys. B441, 234-248 (1995), hep-ph/9412283

[23] J. Levelt, and P. J. Mulders, Phys. Lett. B338, 357-362 (1994), hep-ph/9408257

[24] M. Burkardt, hep-ph 0807.2599 (2008), 0807.2599. 